See Article page 433.

\section{Commentary: Not a "checkmate," but great progress}

\author{
Brendon M. Stiles, MD, and Marc Vimolratana, MD
}

Esophageal cancer continues to be one of our greatest surgical and oncologic challenges. Only a minority of patients will experience a complete pathologic response with combined chemotherapy and radiation therapy before surgery. Those patients with residual disease have recurrence rates as high as $70 \%$ to $75 \%$. Although there has been progress in the neoadjuvant space, the adjuvant space has been notably barren of success stories. However, the recent publication of the CheckMate 577 trial and the subsequent approval by the Food and Drug Administration of adjuvant nivolumab is a game changer for patients with surgically resected esophageal/gastroesophageal cancer with complete resection but residual cancer. ${ }^{1}$

Cameron and colleagues ${ }^{2}$ do an excellent job describing the CheckMate 577 trial and how it easily fits into the typical paradigm of treatment for these patients. The results of the trial were outstanding, with a $31 \%$ reduction (hazard ratio [HR], 0.69; confidence interval [CI], 0.560.86 ) in the risk of recurrence/death and a doubling of disease-free survival (DFS; 22.4 vs 11.0 months) for patients randomized to nivolumab. We agree that adjuvant nivolumab has little impact for surgeons on the perioperative care of their patients, making this approach quite attractive. The allowance of randomization up to 16 weeks after surgery allows patients the time to recover from the unique physiological challenges of esophagectomy. We also agree with the authors that the inclusion of patient-reported

\footnotetext{
From the Department of Cardiothoracic and Vascular Surgery, Montefiore Health System, Albert Einstein College of Medicine, Bronx, NY.

Disclosures: Dr Stiles reported consulting fees from AstraZeneca, Pfizer, Flame Biosciences, and Gala/Galvanize Therapeutics; advisory boards for Bristol Myers Squibb and Genentech; and board member of the Lung Cancer Research Foundation. Dr Vimolratana reported no conflicts of interest.

The Journal policy requires editors and reviewers to disclose conflicts of interest and to decline handling or reviewing manuscripts for which they may have a conflict of interest. The editors and reviewers of this article have no conflicts of interest.

Received for publication Aug 29, 2021; revisions received Aug 29, 2021; accepted for publication Aug 31, 2021; available ahead of print Sept 8, 2021.

Address for reprints: Brendon M. Stiles, MD, Department of Cardiothoracic and Vascular Surgery Montefiore Health System, Albert Einstein College of Medicine, 4th floor, 111 E 210th St, Bronx, NY 10467 (E-mail: brstiles@montefiore.org).

J Thorac Cardiovasc Surg 2022;164:438-9

$0022-5223 / \$ 36.00$

Copyright (c) 2021 by The American Association for Thoracic Surgery

https://doi.org/10.1016/j.jtcvs.2021.08.076
}

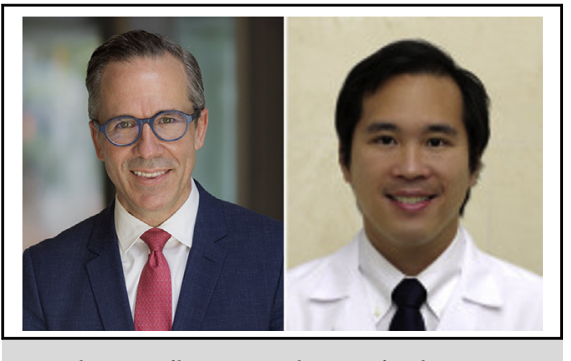

Brendon M. Stiles, MD, and Marc Vimolratana, MD

CENTRAL MESSAGE

Adjuvant nivolumab improves disease-free survival in patients with surgically resected esophageal and gastroesophageal junction cancers, but patient selection remains important.

outcomes is critical in adjuvant trials. Improvements were seen from baseline scores and there were essentially no differences between patients receiving nivolumab and placebo. That, combined with the low rate of adverse events in the nivolumab arm, makes an easy case for patients.

Where we disagree a bit with the authors is on the "lack of dependency on PD-L1 expression" and the blanket "broad applicability." Given the context of the approval from the Food and Drug Administration, nivolumab should certainly be considered for all eligible patients. However, although CheckMate 577 was stratified by tumor cell programmed death-ligand 1 expression, esophageal/gastric cancers are typically classified by the combined positive score (CPS), a measure of tumor and inflammatory cells. A post-hoc analysis showed that patients with a CPS of at least 5 $(\mathrm{n}=371)$ had markedly improved DFS (HR, 0.62; CI, 0.46-0.83; median DFS, 29.4 vs 10.2 months vs placebo) compared with patients $(\mathrm{n}=295)$ with CPS $<5$ (HR, 0.89; CI, 0.65-1.22; median DFS, 16.3 vs 11.1 months vs placebo). A similar dependence of response on CPS has been noted in the CheckMate 649 study in metastatic gastroesophageal cancer. ${ }^{3}$ Future studies are needed to better define biomarkers to identify subgroups of patients that will truly benefit from adjuvant nivolumab.

It is also important to note some of the caveats of any adjuvant approach. Outside of clinical trials, many patients don't make it to or are unable to tolerate adjuvant therapy after esophagectomy. This is true even of healthier trial patients in the MAGIC (Medical Research Council 
Adjuvant Gastric Infusional Chemotherapy) and FLOT-4 (5-Fluorouracil, Leucovorin, Oxaliplatin and Docetaxel) trials. ${ }^{4,5}$ Because CheckMate 577 only enrolled after trimodality therapy was completed, we have no idea how many patients will drop out in the real world and not receive adjuvant nivolumab. A neoadjuvant immunotherapy approach may therefore be preferable, similar to CheckMate 816 in lung cancer.

Nevertheless, CheckMate 577 is a major breakthrough for patients with surgically resectable esophageal/gastroesophageal junction cancer. With a host of ongoing trials as described by Cameron and colleagues, the pieces on the chessboard are moving rapidly. However, CheckMate 577 certainly gets us closer to the elusive endgame of cure for this deadly disease.

\section{References}

1. Kelly RJ, Ajani JA, Kuzdzal J, Zander T, Van Cutsem E, Piessen G, et al. Adjuvan nivolumab in resected esophageal or gastroesophageal junction cancer. $N$ Engl J Med. 2021;384:1191-203.

2. Cameron RB, Ajani JA, Wu AJ. Adjuvant nivolumab following preoperative chemoradiotherapy and surgery in esophageal cancer: a shifting paradigm. $J$ Thorac Cardiovasc Surg. 2022;164:433-7.

3. Janjigian YY, Shitara K, Moehler M, Garrido M, Salman P, Shen L, et al. First-line nivolumab plus chemotherapy versus chemotherapy alone for advanced gastric, gastro-oesophageal junction, and oesophageal adenocarcinoma (CheckMate 649): a randomized, open-label, phase 3 trial. Lancet. 2021;398:27-40.

4. Cunningham D, Allum WH, Stenning SP, Thompson JN, Van de Velde CJ, Nicolson M, et al. Perioperative chemotherapy versus surgery alone for resectable gastroesophageal cancer. $N$ Engl J Med. 2006;355:11-20.

5. Al-Batran SE, Homann N, Schmalenberg H, Kopp HG, Haag GM, Luley KB, et al Perioperative chemotherapy with docetaxel, oxaliplatin, and fluorouracil/leucovorin (FLOT) versus epirubicin, cisplatin, and fluorouracil or capecitabine $(\mathrm{ECF} / \mathrm{ECX})$ for resectable gastric or gastroesophageal junction (GEJ) adenocarcinoma (FLOT4-AIO): a multicenter, randomized phase 3 trial. J Clin Oncol. 2017; 35(suppl 15):4004. 